TRANSACTIONS OF THE

AMERICAN MATHEMATICAL SOCIETY

Volume 350, Number 7, July 1998, Pages 2737-2752

S $0002-9947(98) 01942-4$

\title{
PICARD GROUPS AND INFINITE MATRIX RINGS
}

\author{
GENE ABRAMS AND JEREMY HAEFNER
}

\begin{abstract}
We describe a connection between the Picard group of a ring with local units $T$ and the Picard group of the unital overring $\operatorname{End}\left({ }_{T} T\right)$. Using this connection, we show that the three groups $\operatorname{Pic}(R), \operatorname{Pic}(F M(R))$, and $\operatorname{Pic}(R F M(R))$ are isomorphic for any unital ring $R$. Furthermore, each element of $\operatorname{Pic}(R F M(R))$ arises from an automorphism of $R F M(R)$, which yields an isomorphsm between $\operatorname{Pic}(\operatorname{RFM}(R))$ and $\operatorname{Out}(\operatorname{RFM}(R))$. As one application we extend a classical result of Rosenberg and Zelinsky by showing that the group $\operatorname{Out}_{R}(R F M(R))$ is abelian for any commutative unital ring $R$.
\end{abstract}

We recall (see e.g. [3]) that a ring $A$ is said to have local units in case $A$ contains a set of idempotents $E$ for which $A=\bigcup_{e \in E} e A e$. In particular, any unital ring has local units. In this article we describe various connections between the Picard group of a ring with local units and the Picard groups of related unital rings. The motivation for this investigation stems from some work of Beattie and del Río [4], which includes an investigation of the Picard group of the category of graded modules over a ring graded by an infinite group. They show that a generalization of the standard exact sequence $1 \rightarrow \operatorname{Inn}(A) \rightarrow \operatorname{Aut}(A) \rightarrow \operatorname{Pic}(A)$ arises, where one must consider automorphisms, inner automorphisms, and invertible bimodules in the more general local units context.

For any ring with local units $A$ the $\operatorname{ring} B=\operatorname{End}\left({ }_{A} A\right)$ is a unital ring into which $A$ embeds (via right multiplications) as a right ideal. (If $A$ itself is unital then $A=B$.) Our goal here is to investigate the relationship between the aforementioned exact sequence and the exact sequence $1 \rightarrow \operatorname{Inn}(B) \rightarrow \operatorname{Aut}(B) \rightarrow \operatorname{Pic}(B)$.

In Section 1 we show that there is an isomorphism between a subgroup $J$ of $\operatorname{Pic}(A)$ and a subgroup $H$ of $\operatorname{Pic}(B)$. In Section 2 we apply this isomorphism in the specific case where $A=F M(R)$ (the ring of countably infinite matrices with entries from the unital ring $R$, whose elements have almost all components equal to 0 ). In this situation we get $B=R F M(R)$, the (unital) ring of row-finite matrices over $R$. We show that $J=\operatorname{Pic}(F M(R))$ and $H=\operatorname{Pic}(R F M(R))$, and each in turn is isomorphic to $\operatorname{Pic}(R)$. We discuss how this result can be interpreted in the context of recent work by Guralnick and Montgomery [11]. We then apply this isomorphism to conclude that the group $\mathrm{Out}_{R}(R F M(R))$ of outer automorphisms of $R F M(R)$ which fix $R$ elementwise is abelian whenever $R$ is a commutative unital ring. This extends the well-known result of Rosenberg and Zelinsky [12, Corollary $6]$ to the infinite matrix setting.

Throughout this paper $A$ will denote a ring with local units with set of idempotents $E ; B$ will denote $\operatorname{End}\left({ }_{A} A\right)$. For each $a \in A$ we have $\rho_{a} \in B$ via $(x) \rho_{a}=x a$;

Received by the editors November 16, 1995.

1991 Mathematics Subject Classification. Primary 16A42, 16A64.

(C) 1998 American Mathematical Society 
the map $\rho: A \rightarrow B$ via $a \mapsto \rho_{a}$ gives an embedding of $A$ in $B$ as a right ideal. We will often identify $a \in A$ with its image $\rho_{a} \in B$ without explicit mention.

A left $A$-module $M$ is called unitary in case $A M=M$; the category $A$-mod is defined to be the collection of unitary left $A$-modules, together with usual homomorphisms. Unless otherwise indicated, the word module (resp. bimodule) will always mean unitary module (resp. unitary bimodule). All module homomorphisms will be written opposite the scalars.

Analogous to the definition for unital rings, the group Pic $(A)$ consists of those $A-A$ bimodules $P$ which are both left and right unitary, and for which there exists a (left and right unitary) $A-A$ bimodule $Q$ having $P \otimes Q \cong A$ and $Q \otimes P \cong A$ as bimodules. The operation in $\operatorname{Pic}(A)$ is $\otimes_{A}$. By [3, Theorem 2.2] $\operatorname{Pic}(A)$ is precisely the group of category autoequivalences of $A$-mod.

If $\phi$ is an automorphism of the $\operatorname{ring} T$ and $X$ is any subset of $T$, we often denote the set $(X) \phi$ by $X^{\phi}$. For $\phi \in \operatorname{Aut}(T)$ we construct the $T-T$ bimodule $T_{\phi}$ which as a set is $T$, has the same left module structure as ${ }_{T} T$, and as a right $T$ module has structure given by setting $t * x=t x^{\phi}$ for $t, x \in T$. It is straightforward to show that even in the more general setting of rings with local units we have $T_{\phi} \in \operatorname{Pic}(T)$ (with inverse $T_{\phi^{-1}}$ ), and the map $\phi \mapsto T_{\phi}$ is a group homomorphism from $\operatorname{Aut}(T)$ to $\operatorname{Pic}(T)$. If $T$ is unital, we say that $\operatorname{Pic}(T)$ is outer induced in case this homomorphism is surjective. (Bolla [5] calls such rings rings having the Aut-Pic property.) The kernel of this homomorphism in the unital ring situation is the subgroup $\operatorname{Inn}(T)$ of inner automorphisms of $T$; thus there is an embedding of the group $\operatorname{Out}(T)=\operatorname{Aut}(T) / \operatorname{Inn}(T)$ into $\operatorname{Pic}(T)$, which is an isomorphism in case $\operatorname{Pic}(T)$ is outer induced. More generally, if $A$ is a ring with local units, Beattie and del Río define $\operatorname{Inn}(A)$ to be the kernel of the homomorphism $\phi \mapsto T_{\phi}$. In this situation the elements of $\operatorname{Inn}(A)$ are explicitly described in [4, Section 1].

Following [10], if $T$ is any ring and $M$ is a left $T$-module then $\operatorname{Div}(M)$ denotes those left $T$-modules $N$ such that $N$ is isomorphic to a direct summand of a finite direct sum of copies of $M$; i.e., those $N$ for which there is a split epimorphism $M^{s} \rightarrow N$ for some integer $s$.

\section{An isomorphism BetWeen Subgroups of $\operatorname{Pic}(A)$ And $\operatorname{Pic}(B)$}

In this section we show that there is a strong connection between the Picard groups $\operatorname{Pic}(A)$ and $\operatorname{Pic}(B)$. Specifically, we show that for any ring with local units $A$ there are subgroups $J$ of $\operatorname{Pic}(A)$ and $H$ of $\operatorname{Pic}(B)$ for which $J \cong H$. We then show (in Section 2 as well as in [1]) that in many situations we have $J=\operatorname{Pic}(A)$ and $H=\operatorname{Pic}(B)$, thus yielding an isomorphism between the two relevant Picard groups.

For any right $A$-module $M_{A}$ we define a right $B$-module structure on $M$ by setting $m * b=m(e b)$, where $e \in E$ has $m e=m$. It is easy to show that this is well-defined. In fact, $M_{B}$ is isomorphic to the tensor product $M \otimes_{A} A_{B}$, via the map $m \otimes a \mapsto m a$ (whose inverse is given by the map $m \mapsto m \otimes e$ where $e \in E$ has $m e=m$ ). In case $M=A_{A}$ this is just right multiplication of $B$ on $A$. Given this, for any $A-A$ bimodule ${ }_{A} X_{A}$ we can define a $B-B$ bimodule structure on

$$
\widehat{X}=\operatorname{Hom}_{A}(A, X)
$$

by viewing $\widehat{X}$ as $\operatorname{Hom}_{A}\left({ }_{A} A_{B},{ }_{A} X_{B}\right)$. Specifically, for $b \in B$ and $f \in \operatorname{Hom}_{A}(A, X)$ we define $b * f=\rho_{b} \circ f$, where $\rho_{b}$ denotes right multiplication by $b$. Similarly, we 
define $f * b$ by setting (for each $a \in A)(a) f * b=(a) f \cdot e b$, where $e \in E$ has (a) $f \cdot e=(a) f$. As abelian groups we always have $X$ embedded in $\widehat{X}$ by $x \mapsto \rho_{x}$; in general this embedding is not surjective. Of course, if $A$ is unital, then $A=B$ and $\rho_{x}$ is an isomorphism for all $x \in X$.

Our immediate goal is to find conditions which ensure that $\widehat{X} \in \operatorname{Pic}(B)$ for $X \in \operatorname{Pic}(A)$. We start with some preliminary results regarding the elements of the Picard group of a ring with local units.

Notation 1.1. (1) If $X$ is an $A-A$ bimodule, then there is a ring homomorphism $\rho^{X}: B \rightarrow \operatorname{End}\left({ }_{A} X\right)$ given by $b \mapsto \rho_{b}^{X}$, where $(x) \rho_{b}^{X}=x * b$.

(2) If $X \in \operatorname{Pic}(A)$, then by definition there exists an $A-A$ bimodule isomorphism $X^{-1} \otimes X \cong A$; we denote this by $\xi^{X}: X^{-1} \otimes X \rightarrow A$.

Proposition 1.2. Suppose $X \in \operatorname{Pic}(A)$. Then $\operatorname{End}\left({ }_{A} X\right) \cong B$ both as rings and as $B-B$ bimodules, via the map $\rho^{X}: B \rightarrow \operatorname{End}\left({ }_{A} X\right)$ which takes $b$ to $\rho_{b}^{X}$.

Proof. Let $C=\operatorname{End}\left({ }_{A} X\right)$. It is clear that $\rho^{X}$ is a ring map, since $\left(b b^{\prime}\right) \rho^{X}=\rho_{b b^{\prime}}^{X}=$ $\rho_{b}^{X} \cdot \rho_{b^{\prime}}^{X}$. We define $\theta^{X}: C \rightarrow B$ by setting $(g) \theta^{X}=\left(\xi^{X}\right)^{-1}\left(1_{X^{-1}} \otimes g\right) \xi^{X}$. Now $\theta^{X}$ is a ring map, as for $g, g^{\prime} \in C$ we have

$$
\begin{gathered}
\left(g g^{\prime}\right) \theta^{X}=\left(\xi^{X}\right)^{-1}\left(1_{X^{-1}} \otimes g g^{\prime}\right) \xi^{X}=\left(\xi^{X}\right)^{-1}\left(1_{X^{-1}} \otimes g\right) \cdot\left(1_{X^{-1}} \otimes g^{\prime}\right) \xi^{X} \\
=\left(\xi^{X}\right)^{-1}\left(1_{X^{-1}} \otimes g\right) \xi^{X} \cdot\left(\xi^{X}\right)^{-1}\left(1_{X^{-1}} \otimes g^{\prime}\right) \xi^{X}=(g) \theta^{X} \cdot\left(g^{\prime}\right) \theta^{X} .
\end{gathered}
$$

We now show that $\rho^{X} \theta^{X}=1_{B}$, which in particular means that $\rho^{X}$ is injective and $\theta^{X}$ is surjective. Let $b \in B$. Then $(b) \rho^{X} \theta^{X}=\left(\rho_{b}^{X}\right) \theta^{X}=\left(\xi^{X}\right)^{-1}\left(1_{X^{-1}} \otimes \rho_{b}^{X}\right) \xi^{X}$. We show that for $a \in A,(a)\left(\xi^{X}\right)^{-1}\left(1_{X^{-1}} \otimes \rho_{b}^{X}\right) \xi^{X}=a b$. But writing $(a)\left(\xi^{X}\right)^{-1}=$ $\sum x_{i}^{\prime} \otimes x_{i}$, where $x_{i}^{\prime} \in X^{-1}$ and $x_{i} \in X$, we have

$$
(a)\left(\xi^{X}\right)^{-1}\left(1_{X^{-1}} \otimes \rho_{b}^{X}\right) \xi^{X}=\left(\sum x_{i}^{\prime} \otimes x_{i}\right)\left(1 \otimes \rho_{b}^{X}\right) \xi^{X}=\left(\sum x_{i}^{\prime} \otimes x_{i} b\right) \xi^{X} .
$$

Since the sum is finite, there exists $e=e^{2} \in A$ such that $a e=a$ and $x_{i} e=x_{i}$ for each $i$. Thus,

$$
\begin{gathered}
(a)\left(\xi^{X}\right)^{-1}\left(1_{X^{-1}} \otimes \rho_{b}^{X}\right) \xi^{X}=\left(\sum x_{i}^{\prime} \otimes x_{i} e b\right) \xi^{X}=\sum\left(x_{i}^{\prime} \otimes x_{i} e b\right) \xi^{X} \\
=\sum\left(x_{i}^{\prime} \otimes x_{i}\right) \xi^{X}(e b)=\left(\sum x_{i}^{\prime} \otimes x_{i}\right) \xi^{X}(e b)=a(e b)=a b
\end{gathered}
$$

where the $e b$ may be factored out since $\xi^{X}$ is a right $A$-map. Thus, for $a \in A$, $(a)\left(\xi^{X}\right)^{-1}\left(1_{X^{-1}} \otimes \rho_{b}^{X}\right) \xi^{X}=a b$, and so

$$
\text { (b) } \rho^{X} \theta^{X}=\left(\rho_{b}^{X}\right) \theta^{X}=\left(\xi^{X}\right)^{-1}\left(1_{X^{-1}} \otimes \rho_{b}^{X}\right) \xi^{X}=\rho_{b}^{A} .
$$

Since the action of $B$ on $A$ is given by right multiplication by the maps $\rho_{b}^{A}$, we see that $\rho_{b}^{A}=b$, and so the claim is shown.

Now suppose $c \in C$ is such that $(c) \theta^{X}=0$. Then $\left(\xi^{X}\right)^{-1}(1 \otimes c) \xi^{X}=0$ in $B$. It follows that $1 \otimes c=0$. But since $X$ is invertible, we see that $c: X \rightarrow X$ is the zero map and so $\theta^{X}$ is injective.

Thus we have that $\theta^{X}$ is a ring isomorphism. To see that $\rho^{X}$ is the inverse of $\theta^{X}$, notice that

$$
\rho^{X}=\rho^{X} \cdot 1_{C}=\rho^{X}\left(\theta^{X}\left[\theta^{X}\right]^{-1}\right)=1_{B}\left[\theta^{X}\right]^{-1}=\left[\theta^{X}\right]^{-1} .
$$

Next we prove that $\rho$ and $\theta$ are bimodule maps. We define a $B-B$ bimodule structure on $C=\operatorname{End}\left({ }_{A} X\right)$ as follows: for $c \in C$ and $b, b^{\prime} \in B, b * c * b^{\prime}:=\rho_{b}^{X} \cdot c \cdot \rho_{b^{\prime}}^{X}$. 
Thus, if $\beta \in B$, then $\left(b \beta b^{\prime}\right) \rho^{X}=\rho_{b}^{X} \rho_{\beta}^{X} \rho_{b^{\prime}}^{X}=b *(\beta) \rho^{X} * b^{\prime}$, so $\rho^{X}$ is a $B-B$ bimodule map.

In a similar manner,

$$
\left(b * c * b^{\prime}\right) \theta^{X}=\left(\rho_{b}^{X} c \rho_{b^{\prime}}^{X}\right)=\left(\rho_{b}^{X}\right) \theta^{X}(c) \theta^{X}\left(\rho_{b^{\prime}}^{X}\right) \theta^{X}=b(c) \theta^{X} b^{\prime} .
$$

Remark 1.3. (1) If $X \in P i c(A)$ and $g \in \operatorname{End}\left({ }_{A} X\right)$, then

$$
(x) g=x *(g) \theta^{X},
$$

where $*$ denotes the induced action of $B$ on $X$. To see this, recall that $\theta^{X} \rho^{X}=$ $1_{\operatorname{End}\left({ }_{A} X\right)}$. Then for each $x \in X$ we have

$$
(x) g=(x) g \circ 1_{\operatorname{End}\left({ }_{A} X\right)}=(x)(g) \theta^{X} \rho^{X}(x) \rho_{(g) \theta^{X}}^{X}=x *(g) \theta^{X} .
$$

(2) The $A-A$ bimodule isomorphism $\xi^{X}: X^{-1} \otimes X \rightarrow A$ satisfies the property

$$
\left(x^{\prime} \otimes(x) g\right) \xi^{X}=\left(x^{\prime} \otimes x\right) \xi^{X}(g) \theta^{X}
$$

for each $x^{\prime} \in X^{-1}, x \in X$, and $g \in \operatorname{End}\left({ }_{A} X\right)$. This is because $\left(x^{\prime} \otimes(x) g\right) \xi^{X}=$ $\left(x^{\prime} \otimes x *(g) \theta^{X}\right) \xi^{X}=\left(x^{\prime} \otimes x * e(g) \theta^{X}\right) \xi^{X}=\left(x^{\prime} \otimes x\right) \xi^{X} e(g) \theta^{X}=\left(x^{\prime} \otimes x\right) \xi^{X}(g) \theta^{X}$.

For a unital ring $C$ the regular module ${ }_{C} C$ is finitely generated; furthermore, if $P \in \operatorname{Pic}(C)$ then ${ }_{C} P$ is finitely generated. These two properties together in turn yield that $P \in \operatorname{Div}(C)$ and $C \in \operatorname{Div}(P)$. For a nonunital ring $A$ with local units the regular module ${ }_{A} A$ is not finitely generated; this implies that an element $X$ of $\operatorname{Pic}(A)$ need not be in $\operatorname{Div}(A)$, and that $A$ need not be in $\operatorname{Div}(X)$; see e.g. Example 1.15 below. We now show that those elements $X$ of $\operatorname{Pic}(A)$ for which $X \in \operatorname{Div}(A)$ and $A \in \operatorname{Div}(X)$ play a key role in our investigation.

Proposition 1.4. Let $X \in \operatorname{Pic}(A)$. Then $\widehat{X} \in \operatorname{Pic}(B)$ if and only if $X \in \operatorname{Div}(A)$ and $A \in \operatorname{Div}(X)$.

Proof. $(\Rightarrow)$ Assume $\widehat{X} \in \operatorname{Pic}(B)$. Then we have in particular that ${ }_{B} \widehat{X}$ is finitely generated and projective. So there exists a split epimorphism of left $B$-modules $\widehat{\alpha}$ : ${ }_{B} B^{n} \rightarrow \widehat{X}$. Denote by $\widehat{\beta}$ a splitting map from $\widehat{X}$ to ${ }_{B} B^{n}$ for $\widehat{\alpha}$; so for each $f \in \widehat{X}$ we have $(f) \widehat{\beta} \widehat{\alpha}=f$.

For $1 \leq i \leq n$ let $f_{i} \in \widehat{X}=\operatorname{Hom}_{A}(A, X)$ denote the image under $\widehat{\alpha}$ of the standard basis element $e_{i}$ of $B^{n}$. Since $\widehat{\alpha}$ is surjective we have that $\left\{f_{1}, \ldots, f_{n}\right\}$ generate $\widehat{X}$ as a left $B$-module. Define $\alpha: A^{n} \rightarrow X$ by setting $\alpha:\left(a_{1}, \ldots, a_{n}\right) \mapsto \sum_{i=1}^{n}\left(a_{i}\right) f_{i}$ for all $\left(a_{1}, \ldots, a_{n}\right) \in A^{n}$. Then it is easy to show that $\alpha$ is a homomorphism of left $A$-modules. Now for $x \in X$ define $(x) \beta=\left(\rho_{x}\right) \widehat{\beta}$. Using the fact that $e x=x$ for some $e \in E$, it is easy to show that $(x) \beta \in A^{n}$. A tedious but straightforward computation yields that $\beta$ splits $\alpha$.

We now show that there is a split epimorphism $\gamma: X^{m} \rightarrow A$ in $A$-mod. But $\widehat{X} \in \operatorname{Pic}(B)$ gives that ${ }_{B} \widehat{X}$ is a generator of $B$-mod. In particular there exists a split epimorphism $\widehat{\gamma}: \widehat{X}^{m} \rightarrow B \rightarrow 0$ in $B$-mod. For $\left(x_{1}, \ldots, x_{m}\right) \in X^{m}$ define $\gamma: X^{m} \rightarrow A$ by setting $\left(x_{1}, \ldots, x_{m}\right) \gamma=\left(\rho_{x_{1}}, \ldots, \rho_{x_{m}}\right) \widehat{\gamma}$. By using methods similar to those above, it is easy to show that $\gamma$ in fact maps into $A$, and that $\gamma$ is left $A$-linear. For $a \in A$ define $(a) \delta=\left((a) f_{1}, \ldots,(a) f_{m}\right) \in X^{m}$, where $\left(f_{1}, \ldots, f_{m}\right) \in \widehat{X}^{m}$ has $\left(f_{1}, \ldots, f_{m}\right) \widehat{\gamma}=1_{A}$. Then $\delta$ is easily shown to split $\gamma$.

$(\Leftarrow)$ Suppose there exist a split epimorphism $\alpha: A^{n} \rightarrow X$ and a split epimorphism $X^{m} \rightarrow A \rightarrow 0$ in $A$-mod. The goal is to show that $\widehat{X} \in \operatorname{Pic}(B)$. We 
do this by showing that there is a Morita context in which $\widehat{X}$ is one of the bimodules. Define the maps $\tau: \widehat{X} \otimes_{B} \operatorname{Hom}_{A}(X, A) \rightarrow \operatorname{Hom}_{A}(A, A)=B$ and $\psi:$ $\operatorname{Hom}_{A}(X, A) \otimes_{B} \widehat{X} \rightarrow \operatorname{Hom}_{A}(X, X)$ by appropriately extending the composition map in each case. Let $\mu$ denote the composition $\psi \circ \theta^{X}: \operatorname{Hom}_{A}(X, A) \otimes_{B} \widehat{X} \rightarrow B$ where $\theta^{X}$ is described in the proof of Proposition 1.2.

Now recall the $B-B$ bimodule action on $\operatorname{Hom}_{A}(X, A)$ and $H_{A}(A, X)$ respectively: for $f \in \operatorname{Hom}_{A}(X, A), g \in \operatorname{Hom}_{A}(A, X)$ and $b, b^{\prime} \in B$, we have

$$
b * f * b^{\prime}:=\rho_{b}^{X} \circ f \circ \rho_{b^{\prime}}^{A} \text { and } b * g * b^{\prime}:=\rho_{b}^{A} \circ g \circ \rho_{b^{\prime}}^{X} .
$$

We show that the maps $\tau$ and $\mu$ satisfy the Morita pair conditions (see e.g. [2, p. 266]); namely, for $h, f \in \operatorname{Hom}_{A}(X, A)$ and $k, g \in \operatorname{Hom}_{A}(A, X)$ we have

$$
\text { (1) }(f \otimes g) \mu * h=f *(g \otimes h) \tau \quad \text { and } \quad(2) \quad k *(f \otimes g) \mu=(k \otimes f) \tau * g .
$$

For (1) we have

$$
\begin{gathered}
(f \otimes g) \mu * h:=\rho_{(f \otimes g) \mu}^{X} \circ h=\rho_{(f \circ g) \theta^{X}}^{X} \circ h=(f \circ g) \theta^{X} \rho^{X} \circ h \\
=(f \circ g) \circ h=(f g) h=f(g h)=f *(g \otimes h) \tau .
\end{gathered}
$$

For (2) we have:

$$
\begin{gathered}
k *(f \otimes g) \mu:=k \circ \rho_{(f \otimes g) \mu}^{X}=k \circ \rho_{(f g) \theta^{X}}^{X}=k \circ[f g] \theta^{X} \rho^{X} \\
=k \circ(f g)=k(f g)=(k f) g=(k \otimes f) \tau * g .
\end{gathered}
$$

Now let $T$ and $W$ be arbitrary rings, and let ${ }_{T} M$ and ${ }_{T} N_{W}$ be modules. Let $f: \operatorname{Hom}_{T}\left(M, N_{W}\right) \otimes_{W} \operatorname{Hom}_{T}\left(N_{W}, M\right) \rightarrow \operatorname{Hom}_{T}(M, M)$ be the extension of the composition map. Suppose there exists a split epimorphism $\nu:{ }_{T} N^{j} \rightarrow{ }_{T} M$ for some integer $j$ (say with splitting map $\chi$ ). Then $f$ is an epimorphism. To see this, let $\pi_{i}: N^{j} \rightarrow N$ be the $i$ th coordinate projection, and let $\iota_{i}: N \rightarrow N^{j}$ be the $i$ th coordinate inclusion. So $\sum \pi_{i} \circ \iota_{i}$ is the identity on $N^{j}$. Now let $\alpha \in \operatorname{Hom}_{T}(M, M)$. Then for each $i$ we have that $\alpha \circ \chi \circ \pi_{i} \in \operatorname{Hom}_{T}(M, N)$, and $\iota_{i} \circ \nu \in \operatorname{Hom}_{T}(N, M)$. We compute:

$$
\begin{gathered}
\left(\sum_{i=1}^{j} \alpha \circ \chi \circ \pi_{i} \otimes \iota_{i} \circ \nu\right) f=\sum_{i=1}^{j} \alpha \circ \chi \circ \pi_{i} \circ \iota_{i} \circ \nu=\alpha \circ \chi \circ\left(\sum_{i=1}^{j} \pi_{i} \circ \iota_{i}\right) \circ \nu \\
=\alpha \circ \chi \circ 1_{N^{j}} \circ \nu=\alpha \circ \chi \circ \nu=\alpha \circ 1_{N}=\alpha .
\end{gathered}
$$

For the specific case at hand, the result of the preceding paragraph, together with the hypotheses, yield that both $\tau$ and $\mu$ are epimorphisms. Therefore the equivalence data $\left(B, B, \widehat{X}, \operatorname{Hom}_{A}(X, A), \tau, \mu\right)$ has both maps surjective, hence yields a Morita equivalence (see e.g. [2, Section 22]). Specifically, this means that tensoring by $\widehat{X}$ and by $\operatorname{Hom}_{A}(X, A)$ each induces a category equivalence from $B$-mod to itself; i.e., that $\widehat{X}$ is an element of $\operatorname{Pic}(B)$.

We note that the proof of the above proposition in fact yields

Corollary 1.5. Suppose $X \in \operatorname{Pic}(A)$ has $\widehat{X} \in \operatorname{Pic}(B)$. Then $\widehat{X}^{-1}=\operatorname{Hom}_{A}(X, A)$. In particular, $\operatorname{Hom}_{A}(X, A) \in \operatorname{Pic}(B)$. 
With Proposition 1.4 in mind, we define

$$
J=\{X \in \operatorname{Pic}(A) \mid X \in \operatorname{Div}(A) \text { and } A \in \operatorname{Div}(X)\} .
$$

Of course, if $A$ is unital then $J=\operatorname{Pic}(A)$. In general, however, $J$ may indeed be proper in $\operatorname{Pic}(A)$; see Example 1.15 below. We now show that the map $X \mapsto \widehat{X}$ is multiplicative; this will consequently give that $J$ is a subgroup of $\operatorname{Pic}(A)$. We start by recording some useful properties.

Lemma 1.6. (1) Let $W$ be any left $B$-module. Then $A W=\left\{\sum a_{i} w_{i} \mid a_{i} \in A\right.$, $\left.w_{i} \in W\right\}$ is the largest (unitary) $A$-submodule of $W$. Moreover, $A \otimes W \cong A W$ as left $A$-modules via the map $\mu$ where $(a \otimes w) \mu=a w$. Similar statements hold for right $B$-modules.

(2) Let $V$ be any left $A$-module. Then $A \otimes_{B} H_{o m}(A, V) \cong V$ as left $A$-modules. In addition, if $V_{C}$ has a right module structure for some ring $C$, then this isomorphism is also a right $C$-module isomorphism.

(3) $\operatorname{Hom}_{A}\left(A, A \otimes_{B} \operatorname{Hom}_{A}(A, V)\right) \cong \operatorname{Hom}_{A}(A, V)$ as left B-modules. In addition, if $V_{C}$ has a right module structure for some ring $C$, then this isomorphism is also a right $C$-module isomorphism.

(4) Let $M$ be a $D-B$ bimodule, and let $N$ be a $B-C$ bimodule (where $C$ and $D$ are arbitrary rings). Then $M A \otimes_{B} N \cong M A \otimes_{A} A N$ as $D-C$ bimodules. In particular, for any $D-A$ bimodule $M, M \otimes_{B} N \cong M \otimes_{A} A N$ as $D-C$ bimodules.

Proof. (1) The first statement is clear. Define $\zeta: A W \rightarrow A \otimes_{B} W$ by setting $\left(\sum a_{i} w_{i}\right) \zeta=e \otimes \sum a_{i} w_{i}$, where $e \in E$ has $e \sum a_{i} w_{i}=\sum a_{i} w_{i}$. It is easy to show that $\zeta$ is well defined, a left $A$-homomorphism, and the inverse of $\mu$.

(2) $A \otimes_{B} H_{o m}(A, V) \cong A H \operatorname{som}_{A}(A, V)$ by part (1). But $A H o m_{A}(A, V) \cong V$ as left $A$-modules by [3, Proposition 1.1]. The isomorphism clearly preserves right structures.

(3) That these are isomorphic as abelian groups follows from (2). But the induced map from $H_{o m}\left(A, A \otimes_{B} \operatorname{Hom}_{A}(A, V)\right)$ to $\operatorname{Hom}_{A}(A, V)$ clearly preserves the left $B$-action, as well as any right action on $V$.

(4) It is easy to show that the map $m \otimes_{B} n \mapsto m \otimes_{A} n$ is an isomorphism of the desired type.

Lemma 1.7. Let $P$ be any finitely generated projective left $B$-module (i.e. $P \in$ $\operatorname{Div}(B))$.

(1) $\widehat{A P}=\operatorname{Hom}_{A}\left(A, A \otimes_{B} P\right)$ is isomorphic to $P$ as left $B$-modules. If $P_{C}$ is also a right $C$-module for some ring $C$, then this isomorphism is as $B-C$ bimodules.

(2) If ${ }_{A} U \in \operatorname{Div}(A)$, then the homomorphism

$$
\eta: \operatorname{Hom}_{A}(U, A) \otimes_{B} P \rightarrow \operatorname{Hom}_{A}\left(U, A \otimes_{B} P\right)
$$

given by setting $(u)[(\gamma \otimes p) \eta]=(u) \gamma \otimes p$ for each $u \in U$ is an isomorphism of abelian groups. This isomorphism is a $D-C$ bimodule isomorphism whenever we have right module structures $U_{D}$ and $P_{C}$ for rings $C$ and $D$.

(3) If ${ }_{A} X_{B}$ is a bimodule (e.g. if $X \in \operatorname{Pic}(A)$ and $X$ is given the right $B$ structure described above), then the homomorphism $\eta: \operatorname{Hom}_{A}(A, X) \otimes_{B} P \rightarrow$ $H_{o m}\left(A, X \otimes_{B} P\right)$ given by setting $(a)[(\gamma \otimes p) \eta]=(u) \gamma \otimes p$ for each $a \in A$ is an isomorphism of abelian groups. This isomorphism is a $D-C$ bimodule isomorphism whenever we have right module structures $A_{D}$ and $P_{C}$ for rings $C$ and D. 
Proof. For each of the three statements we will invoke the idea described in $[2$, Proposition 20.10]. Specifically, if a natural transformation $\zeta$ has the property that $\zeta_{M}$ is an isomorphism for some module $M$, then $\zeta_{N}$ is an isomorphism for any $N \in \operatorname{Div}(M)$.

(1) Let $F$ denote the functor $F: B$-mod $\rightarrow B$-mod given by

$$
F(M)=H_{\text {om }}\left(A_{B}, A \otimes_{B} M\right),
$$

and let $I: B$-mod $\rightarrow B$-mod denote the identity functor. For each $m \in M$ let $\bar{\rho}_{m}: A \rightarrow A \otimes_{B} M$ denote the map given by $(a) \bar{\rho}_{m}=a \otimes m$; then $\bar{\rho}_{m} \in F(M)$. Now let $\xi: I \rightarrow F$ denote the transformation given by setting, for each $M \in B$-mod and each $m \in M,(m) \xi_{M}=\bar{\rho}_{m}$. It is easy to check that $\xi$ is a natural transformation.

We claim that $\xi_{B}: B \rightarrow \operatorname{Hom}_{A}\left(A_{B}, A \otimes_{B} B\right)$ is an isomorphism of abelian groups. But this is clear: if $b \in B$ has $a \otimes b=0$ for all $a \in A$ then $b=0$ (since $A \otimes B \cong A$ as $A-B$ bimodules, and the annihilator in $B$ of $A$ is zero). Similarly, any $A$-homomorphism from $A$ to $A \otimes B$ is just right multiplication by some element of $B$, since $A \otimes B \cong A B \cong A$ as left $A$-modules by Lemma 1.6(1).

But as $\xi$ is a natural transformation we can use the fact that $\xi_{B}$ is an isomorphism together with the aforementioned property to conclude that $\xi_{P}$ is an isomorphism of abelian groups whenever $P \in \operatorname{Div}(B)$. The computation $(a)(b p) \xi_{P}=(a) \bar{\rho}_{b p}=$ $a \otimes b p=a b \otimes p=(a b) \bar{\rho}_{p}=(a) \rho_{b} \circ \bar{\rho}_{p}=(a) b * \bar{\rho}_{p}=(a) b *(p) \xi_{P}$ (for each $a \in A, b \in B, p \in P$ ) yields that $\xi_{P}$ is in fact a left $B$-module isomorphism. It is clear that the map $\xi_{P}$ also preserves whatever right structure there might be on $P$.

(2) We first consider the particular case where $U=A$ as left $A$-modules. Then this homomorphism is the map $\eta_{A}: \operatorname{Hom}_{A}(A, A) \otimes_{B} P \rightarrow \operatorname{Hom}_{A}\left(A, A \otimes_{B} P\right)$. The domain of $\eta_{A}$ is isomorphic to $B \otimes_{B} P \cong P$, and the codomain is also isomorphic to $P$ by part (1). It can be shown that the map $\eta_{A}$ is the composition of these various isomorphisms. Thus $\eta_{U}: \operatorname{Hom}_{A}(U, A) \otimes_{B} P \rightarrow \operatorname{Hom}_{A}\left(U, A \otimes_{B} P\right)$ is an isomorphism for any $U \in \operatorname{Div}(A)$. By tracing through the appropriate maps, it is easy to show that in fact this is a left $D$-module map whenever we have $U_{D}$, and a right $C$-module map whenever we have $P_{C}$.

(3) The homomorphism $\eta_{B}: \operatorname{Hom}_{A}(A, X) \otimes_{B} B \rightarrow \operatorname{Hom}_{A}\left(A, X \otimes_{B} B\right)$ analogous to that described in part (2) is an isomorphism; in fact, each of the appropriate modules is isomorphic to $\operatorname{Hom}_{A}(A, X)$. The result now follows as above.

Proposition 1.8. Let $X, Y \in J$. Then as $B-B$ bimodules we have

(1) $\widehat{X \otimes_{A} Y} \cong \widehat{X} \otimes_{B} \widehat{Y}$, and

(2) $\widehat{X^{-1}} \cong \widehat{X}^{-1}$.

Proof. (1) As $Y \in J$, we have by Proposition 1.4 that $\widehat{Y}=\operatorname{Hom}_{A}(A, Y)$ is in $\operatorname{Pic}(B)$, so ${ }_{B} \widehat{Y}$ is finitely generated projective. Thus Lemma 1.7(3) applies to give the isomorphism

$$
\begin{aligned}
\eta: \widehat{X} \otimes_{B} \widehat{Y}= & \operatorname{Hom}_{A}(A, X) \otimes_{B} \operatorname{Hom}_{A}(A, Y) \\
& \rightarrow \operatorname{Hom}_{A}\left(A, X \otimes_{B} \operatorname{Hom}_{A}(A, Y)\right) .
\end{aligned}
$$

But ${ }_{A} X_{B}={ }_{A} X_{A} \otimes A_{B}$ as $A-B$ bimodules by the remark made at the beginning of this section, so $X \otimes_{B} \operatorname{Hom}_{A}(A, Y) \cong X_{A} \otimes A_{B} \otimes_{B} \operatorname{Hom}_{A}(A, Y) \cong X_{A} \otimes Y$ (by Lemma 1.6(2)). Thus we have an isomorphism

$$
\kappa: \operatorname{Hom}_{A}\left(A, X \otimes_{B} \operatorname{Hom}_{A}(A, Y)\right) \rightarrow \operatorname{Hom}_{A}\left(A, X \otimes_{A} Y\right)=\widehat{X \otimes_{A} Y} .
$$


The composition $\eta \kappa$ provides the desired isomorphism, as it is easily checked that $\eta \kappa$ preserves both the left and right $B$-structures of the appropriate modules.

(2) Let $Y$ denote $X^{-1}$ in $\operatorname{Pic}(A)$; so $X \otimes Y \cong A$ and $Y \otimes X \cong A$. As $X \in J$ we have split epimorphisms of left modules $X^{n} \rightarrow A$ and $A^{t} \rightarrow X$ for some integers $n, t$. Since tensoring preserves split epimorphisms, tensoring each of these maps by $Y$ on the left yields split epimorphisms $Y \otimes X^{n} \rightarrow Y \otimes A$ and $Y \otimes A^{t} \rightarrow Y \otimes X$, which by the standard isomorphisms involving tensor products and direct sums yields split epimorphisms $A^{n} \rightarrow Y$ and $Y^{t} \rightarrow A$. Thus $X^{-1} \in J$. So by Proposition 1.4 we have $\widehat{X^{-1}}=\operatorname{Hom}_{A}\left(A, X^{-1}\right) \in \operatorname{Pic}(B)$. So we may apply Lemma $1.7(3)$ above to get an isomorphism

$$
\operatorname{Hom}_{A}(A, X) \otimes_{B} \operatorname{Hom}_{A}\left(A, X^{-1}\right) \rightarrow \operatorname{Hom}_{A}\left(A, X \otimes_{B} \operatorname{Hom}_{A}\left(A, X^{-1}\right)\right) .
$$

But $X \otimes_{B} \operatorname{Hom}_{A}\left(A, X^{-1}\right) \cong A$ as $A-B$ bimodules, since each of these expressions yields $X^{-1}$ upon tensoring both sides on the left by the invertible $A-A$ bimodule $X^{-1}$. Thus we have an isomorphism $\operatorname{Hom}_{A}\left(A, X \otimes_{B} \operatorname{Hom}_{A}\left(A, X^{-1}\right)\right) \rightarrow$ $\operatorname{Hom}_{A}(A, A)=B$, which is easily shown to be a $B-B$ bimodule isomomorphsm. The composition of these two isomorphisms shows that

$$
\operatorname{Hom}_{A}(A, X) \otimes_{B} \operatorname{Hom}_{A}\left(A, X^{-1}\right) \cong B .
$$

A virtually identical argument yields that $\operatorname{Hom}_{A}\left(A, X^{-1}\right) \otimes_{B} \operatorname{Hom}_{A}(A, X) \cong B$ as well. Thus we have shown that $\widehat{X^{-1}} \cong \widehat{X}^{-1}$, as required.

Proposition 1.9. $J$ is a subgroup of $\operatorname{Pic}(A)$, and the map $X \mapsto \widehat{X}$ is a group homomorphism from $J$ to $\operatorname{Pic}(B)$.

Proof. Let $X, Y \in J$. Then $\widehat{X}$ and $\widehat{Y}$ are in $\operatorname{Pic}(B)$ by Proposition 1.4, so $\widehat{X} \otimes \widehat{Y} \in \operatorname{Pic}(B)$. With Proposition 1.8(1) this yields that $\widehat{X \otimes Y} \in \operatorname{Pic}(B)$, so that $X \otimes Y \in J$ (again by Proposition 1.4). Thus $J$ is closed under products. Clearly $A \in J$. Finally, it was shown in the proof of Proposition 1.8(2) that $J$ is closed under inverses.

The second statement follows from Proposition 1.8(1).

We now identify the corresponding subgroup of $\operatorname{Pic}(B)$.

Definition 1.10. Let $A$ be a ring with local units, and let $B=\operatorname{End}\left({ }_{A} A\right)$. We define

$$
\begin{aligned}
H= & \left\{P \in P i c(B) \mid A P \text { and } A P^{-1} \text { are unitary right } A \text {-modules }\right\} \\
& =\left\{P \in P i c(B) \mid A P=A P A \text { and } A P^{-1}=A P^{-1} A\right\} .
\end{aligned}
$$

Analogous to the situation relating $J$ to $\operatorname{Pic}(A)$, we of course have that $H=$ $\operatorname{Pic}(B)$ whenever $A$ is unital (since then $A=B$ ), but that $H$ may be proper in $\operatorname{Pic}(B)$ more generally (see Example 1.18 below).

Proposition 1.11. $H$ is a subgroup of Pic $(B)$, and the map $P \mapsto A P$ is a group homomorphism from $H$ to $\operatorname{Pic}(A)$.

Proof. Clearly $B \in H$ (as $A B=A$ is right unitary), and $H$ is closed under inverses by definition. If $P, Q \in H$ then we want to show that $A\left(P \otimes_{B} Q\right)$ is right $A$-unitary. But $A\left(P \otimes_{B} Q\right)=A P \otimes_{B} Q=A P A \otimes_{B} Q \cong A P A \otimes_{A} A Q$ (by Lemma 1.6(4)) $=A P A \otimes_{A} A Q A$. Since the isomorphism is as $A-B$ bimodules, and the last module is right $A$-unitary, so is $A\left(P \otimes_{B} Q\right)$. The second statement follows directly from this same computation; namely, that $A\left(P \otimes_{B} Q\right) \cong A P A \otimes_{A} A Q=A P \otimes_{A} A Q$. 
Proposition 1.12. Let $P \in H$. Then $A P \in J$.

Proof. We first show that $A P \in P i c(A)$. But $A P$ and $A P^{-1}$ are each unitary (left and) right $A$-modules by hypothesis; that is, $A P=A P A$ and $A P^{-1}=A P^{-1} A$. Now $A P \otimes_{A} A P^{-1}=A P A \otimes_{A} A P^{-1}=A P A \otimes_{B} P^{-1}$ (by Lemma 1.6(4)) $A P \otimes_{B} P^{-1} \cong A B=A$. Similarly one can show that $A P^{-1} \otimes_{A} A P \cong A$. Thus $A P \in \operatorname{Pic}(A)$; in fact, $A P^{-1} \in \operatorname{Pic}(A)$, and $(A P)^{-1}=A P^{-1}$. We now must show that $A P \in \operatorname{Div}(A)$ and $A \in \operatorname{Div}(A P)$. But as $B$ is unital, we have split epimorphisms of left $B$-modules $B^{n} \rightarrow P$ and $P^{t} \rightarrow B$ for some integers $n$, $t$. On tensoring each of these on the left by $A_{B}$ and using the standard isomorphisms we get the desired properties.

Proposition 1.13. Let $X \in J$. Then $\widehat{X} \in H$.

Proof. By Proposition 1.4 we have that $\widehat{X} \in P i c(B)$. It suffices then to show that $A \widehat{X}$ and $A \widehat{X}^{-1}$ are right $A$-unitary. By Lemma 1.6(2) we have $A \widehat{X} \cong X$ as $A-A$ bimodules, so that $A \widehat{X}$ is right $A$-unitary. By Proposition $1.8(2)$ we have that $\widehat{X^{-1}} \cong \widehat{X}^{-1} ;$ so $A \widehat{X}^{-1} \cong A \widehat{X}^{-1} \cong X^{-1}$ (again by Lemma $1.6(2)$ ), whence $A \widehat{X}^{-1}$ is also right $A$-unitary. Thus $\widehat{X} \in H$.

Finally we are in position to prove the main result of this section.

Theorem 1.14. Let $A$ be a ring with local units, and let $B=\operatorname{End}\left({ }_{A} A\right)$. Then $J \cong H$. In particular, the maps $X \mapsto \widehat{X}$ and $P \mapsto A P$ give the appropriate inverse group isomorphisms.

Proof. With the results of the previous four propositions in mind, the second statement is all that remains to be verified. But for $X \in J$ we have $A \widehat{X} \cong X$ by Lemma 1.6(2). Furthermore, for $P \in H$ we have $\widehat{A P} \cong P$ by Lemma 1.7(1). This completes the proof of the theorem.

We now give an example of a ring with local units $A$ for which $J \neq \operatorname{Pic}(A)$.

Example 1.15. For each positive integer $i$ let $R_{i}$ denote a unital ring for which there exists an element $P_{i}$ of $\operatorname{Pic}(R)$ with the property that $P_{i}$ is generated by no less than $i$ elements as a left $R_{i}$-module. For instance, let $k$ be a field, let $R_{i}=M_{i}(k) \oplus k$, and let $P_{i}$ be the natural $k$-complement of $R_{i}$ when viewed inside $M_{i+1}(k)$. (For a specific description of the case $i=2$ see Example 2.12 below.) As another example, $R_{i}$ can be taken to be an integral domain as constructed in [9]. Let $A$ denote the ring direct sum $\bigoplus_{i \in \mathbf{N}} R_{i}$. Then $A$ is a ring with local units. Moreover, $P=\bigoplus_{i \in \mathbf{N}} P_{i}$ is an $A-A$ bimodule coordinatewise. Using the fact that tensor products distribute over direct sums, it is easy to see that $P \in \operatorname{Pic}(A)$, as $P^{-1}=\bigoplus_{i \in \mathbf{N}} P_{i}^{-1}$. But $P \notin \operatorname{Div}(A)$, as no finite direct sum of copies of $A$ can generate all of $P$. Thus $P \notin J$. (In fact, in the matrix example of the $R_{i}$ we may also conclude that $A \notin \operatorname{Div}(P)$ as well.)

In Example 1.18 below we present a situation in which $H \neq \operatorname{Pic}(B)$. To justify this example we first need some properties of automorphisms of $B$; recall the notation pertaining to these given in the introduction.

Lemma 1.16. Let $A$ be a ring with local units, and let $B=\operatorname{End}\left({ }_{A} A\right)$. Let $\sigma \in$ Aut $(B)$, so that $B_{\sigma} \in \operatorname{Pic}(B)$. Then $A B_{\sigma}$ is right $A$-unitary if and only if $A \subseteq$ $B \cdot A^{\sigma}$. 
Proof. $(\Leftarrow)$ As sets we have $A B_{\sigma}=A$, and the right $A$-action is given by setting $a * x=a x^{\sigma}$. So it suffices to show that for $a \in A$ there exists $g \in A$ with $a g^{\sigma}=a$. There exists $e \in E$ with $a e=a$. The hypotheses yield that there exists $f \in B A$ with $f^{\sigma}=e$. So $a f^{\sigma}=a$. But $f \in B A$ implies there is $g \in A$ with $f g=f$. Then $a g^{\sigma}=\left(a f^{\sigma}\right) g^{\sigma}=a(f g)^{\sigma}=a f^{\sigma}=a$.

$(\Rightarrow)$ Suppose for each $a \in A$ there exists $e \in A$ with $a=a * e=a e^{\sigma}$. But then $a=a e^{\sigma} \in B \cdot A^{\sigma}$.

Since $\left(B_{\sigma}\right)^{-1}=B_{\sigma^{-1}}$ in $\operatorname{Pic}(B)$, we get, as an immediate consequence,

Corollary 1.17. Let $A$ be a ring with local units, let $B=\operatorname{End}\left({ }_{A} A\right)$, and let $\sigma \in$ Aut $(B)$. Then the element $B_{\sigma}$ of Pic $(B)$ is in $H$ if and only if $A \subseteq B \cdot A^{\sigma}$ and $A \subseteq B \cdot A^{\sigma^{-1}}$.

The following example was suggested by Juan Jacobo Simón.

Example 1.18. Let $k$ be a field, let $T=F M(k)$, and let $R=R F M(k)$. Let $A=R \times T$, the ring direct product of $R$ and $T$. Then we easily get $B=\operatorname{End}\left({ }_{A} A\right)=$ $R \times R$. Let $\phi \in \operatorname{Aut}(B)$ be the transpose automorphism, given by $(x, y) \phi=(y, x)$. Then $A^{\phi}=T \times R$, so that $B \cdot A^{\phi}=R T \times R$. But $A=R \times T$ is not contained in $B \cdot A^{\phi}=R T \times R$. We now apply Corollary 1.17 to conclude that the element $B_{\phi}$ of $\operatorname{Pic}(B)$ is not in $H$.

We take up in [1] the issue of providing sufficient conditions on $A$ and/or $B$ which imply that $J=\operatorname{Pic}(A)$ and/or $H=\operatorname{Pic}(B)$. In addition, we provide examples in which the Picard groups $\operatorname{Pic}(A)$ and $\operatorname{Pic}(B)$ are not isomorphic.

\section{2. $\operatorname{Pic}(R)$ Is Isomorphic to $\operatorname{Pic}(\operatorname{RF} M(R))$}

The main result of this second section is that, for any unital ring $R$, the groups $\operatorname{Pic}(R)$ and $\operatorname{Pic}(R F M(R))$ are isomorphic. In particular, we conclude that for any unital ring $R$ there is a unital ring $B$ with $\operatorname{Pic}(B)$ outer induced for which $\operatorname{Pic}(R) \cong \operatorname{Pic}(B)$. We will apply this isomorphism to prove an extension of $[12$, Corollary 6] to infinite matrix rings; specifically, we prove that $\operatorname{Out}_{R}(R F M(R))$ is an abelian group for any unital commutative ring $R$.

We start by providing a general context for these results. Let $R$ be any unital ring and $n$ any positive integer. Following [11], we define the subgroup $I_{n}$ of $\operatorname{Pic}(R)$ by setting $I_{n}=\left\{X \in \operatorname{Pic}(R) \mid X^{n} \cong R^{n}\right.$ as left $R$-modules $\}$. By [8, Theorem $55.12], I_{1}$ is the image of $\operatorname{Out}(R)$ in $\operatorname{Pic}(R)$. More generally, in fact, there is an isomorphism of groups $\alpha_{n}: \operatorname{Out}\left(M_{n}(R)\right) \rightarrow I_{n}$. In addition, there is an embedding $\operatorname{Out}\left(M_{n}(R)\right) \subseteq \operatorname{Out}(R F M(R))$. Our main result implies that there is a group isomorphism $\alpha: \operatorname{Out}(\operatorname{RFM}(R)) \rightarrow \operatorname{Pic}(R)$ for which the diagram

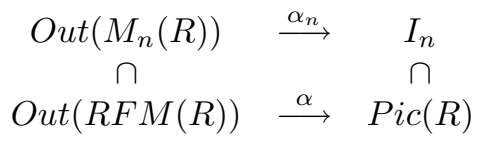

commutes. We note that the group $\operatorname{Out}(R F M(R))$ (resp. Pic $(R))$ is in general too large to be the direct union of the subgroups $\operatorname{Out}\left(M_{n}(R)\right)$ (resp. $\left.I_{n}\right)$.

That an isomorphism between the groups $\operatorname{Out}(\operatorname{RFM}(R))$ and $\operatorname{Pic}(R)$ should exist is in part due to a result of Eilenberg (see e.g. [6]), which yields that ${ }_{R} P^{(\mathbf{N})} \cong$ ${ }_{R} R^{(\mathbf{N})}$ for any $P \in \operatorname{Pic}(R)$. This suggests that the subgroup $I_{\mathbf{N}}$ of $\operatorname{Pic}(R)$ might naturally be defined to be all of $\operatorname{Pic}(R)$; from this perspective, the isomorphism 
Out $(R F M(R)) \rightarrow \operatorname{Pic}(R)$ may then be viewed as the 'infinite extension' of the isomorphisms Out $\left(M_{n}(R)\right) \rightarrow I_{n}$ described above.

The ring $T$ is said to have $S B N$ (for single basis number) provided $T^{n} \cong T^{m}$ as left $T$-modules for all positive integers $n, m$. It is easy to show that this condition is equivalent to the existence of a left $T$-module isomorphism $T \cong T \oplus T$.

Lemma 2.1. Let $A$ be a ring with local units, and let $B=\operatorname{End}\left({ }_{A} A\right)$. Then $A$ has $S B N$ if and only if $B$ has $S B N$.

Proof. Suppose $A$ has SBN; so there are maps $\alpha: A \times A \rightarrow A$ and $\beta: A \rightarrow A \times A$ such that $\alpha \beta=1_{A \times A}$ and $\beta \alpha=1_{A}$. We can write

$$
\alpha=\left(\begin{array}{c}
a \\
b
\end{array}\right) \text { and } \beta=\left(\begin{array}{ll}
c & d
\end{array}\right)
$$

where $a, b, c, d \in B, a c=b d=1_{A}, a d=0=b c$, and $a c+b d=1_{A}$. But since these are elements of $B$ as well, then we can view them as right multiplication on $B$; i.e., we have maps $\rho_{\alpha}: B \times B \rightarrow B$ and $\rho_{\beta}: B \rightarrow B \times B$ such that $\rho_{\alpha}$ and $\rho_{\beta}$ are right multiplications by $\alpha$ and $\beta$, respectively. Since the relations above still hold, namely, $\alpha \beta=1$ and $\beta \alpha=1$, we see that $B$ has SBN.

Conversely, suppose there are maps $\alpha: B \times B \rightarrow B$ and $\beta: B \rightarrow B \times B$ such that $\alpha \beta=1_{B \times B}$ and $\beta \alpha=1_{B}$. We can write

$$
\alpha=\left(\begin{array}{c}
a \\
b
\end{array}\right) \text { and } \beta=\left(\begin{array}{ll}
c & d
\end{array}\right),
$$

where $a c=b d=1_{B}, a d=0=b c$, and $a c+b d=1_{B}$. Since $B$ is a ring with identity, the elements $a, b, c, d$ belong to $B$, which means they are endomorphisms of $A$; i.e., $a, b, c, d \in \operatorname{End}\left({ }_{A} A\right)$. It follows that $A$ has $\mathrm{SBN}$.

Lemma 2.2. Let $R$ be a unital ring. Let $A=F M(R)$, and let $B=R F M(R)$.

(1) $B=\operatorname{End}\left({ }_{A} A\right)$ as right multiplications.

(2) Both $A$ and $B$ have $S B N$.

Proof. (1) As $A$ is a right ideal of $B$, right multiplication by an element of $R F M(R)$ is an element of $\operatorname{End}\left({ }_{A} A\right)$. Now for any positive integer $i$ and $f \in \operatorname{End}\left({ }_{A} A\right)$ we have $\left(e_{i i}\right) f=e_{i i}\left(e_{i i}\right) f$. In particular $\left(e_{i i}\right) f$ has all rows equal to zero except the $i^{t h}$. Define the matrix $M$ whose $i^{\text {th }}$ row is the $i^{\text {th }}$ row of $\left(e_{i i}\right) f$. So in fact $M=\sum_{i \in \mathbf{N}}\left(e_{i i}\right) f=$ $\sum_{i \in \mathbf{N}} e_{i i} \cdot\left(e_{i i}\right) f$. Note that $M \in R F M(R)$. But there exists $\left\{i_{1}, \ldots, i_{n}\right\} \subseteq \mathbf{N}$ such that $a=a \sum_{k=1}^{n} e_{i_{k} i_{k}}$. Then $(a) f=\left(a \sum_{k=1}^{n} e_{i_{k i} i_{k}}\right) f=a\left(\sum_{k=1}^{n} e_{i_{k i} i_{k}}\right) f=a M$.

(2) The ring $R F M(R)$ is well-known to have SBN (see e.g. [2, Exercise 8.14]). Now apply part (1) with Lemma 2.1.

Lemma 2.3. Let $T$ be a ring with $S B N$.

(1) For any $P \in P i c(T), P \oplus P \cong P$ as left $T$-modules.

(2) For any element $P \in J \subseteq P i c(T)$ we have ${ }_{T} P \cong{ }_{T} T$. In particular, for any unital $S B N$ ring $R,{ }_{R} P \cong{ }_{R} R$ for any $P \in \operatorname{Pic}(R)$.

Proof. (1) Let $P \in P i c(T)$. We claim that $P \oplus P \cong P$ as left $T$-modules. Let $Q$ denote $P^{-1}$ in $P i c(T)$. Then as left $T$-modules we have

$$
Q \otimes(P \oplus P) \cong(Q \otimes P) \oplus(Q \otimes P) \cong T \oplus T \cong T \cong Q \otimes P,
$$

which with the autoequivalence property of $Q$ yields $P \oplus P \cong P$, as required. We note that this in turn gives $P^{m} \cong P$ for any integer $m$. 
(2) As $P \in J$ we have that $P$ is a direct summand of $T^{n}$ for some integer $n$; this means that $P$ is a direct summand of $T$ (since $T$ has $\mathrm{SBN}$ ); say $T=P \oplus V$. Similarly, $T$ is a direct summand of $P^{m}$ for some integer $m$. But $P^{m}$ is isomorphic to $P$ by the previous paragraph, so $T$ is a direct summand of $P$; say $P=T \oplus U$. So we have left $T$-module isomorphisms

$$
\begin{aligned}
P=T \oplus U & \cong T \oplus T \oplus U \cong T \oplus P \cong P \oplus V \oplus P \\
& \cong P \oplus P \oplus V \cong P \oplus V \cong T .
\end{aligned}
$$

The last statement follows since for unital rings $R$ we always have $J=\operatorname{Pic}(R)$.

We note that we do not know whether part (1) of the previous lemma is valid for an arbitrary progenerator ${ }_{T} P$ of $T$.

Corollary 2.4. Pic $(R F M(R))$ is outer induced for any unital ring $R$. In particular, there is an isomorphism of groups $\tau: \operatorname{Out}(\operatorname{RFM}(R)) \rightarrow \operatorname{Pic}(\operatorname{RFM}(R))$.

Proof. Let $B$ denote $R F M(R)$. By Lemmas 2.2(2) and 2.3 we have that every element $P$ of $P i c(B)$ has ${ }_{B} P \cong{ }_{B} B$. But as $B$ is unital we may apply [8, Theorem $55.12]$ to conclude that every element of $\operatorname{Pic}(B)$ is of the form $B_{\phi}$ for some $\phi \in$ $\operatorname{Aut}(B)$.

Proposition 2.5. Let $R$ be a unital ring. Then $\operatorname{Pic}(R) \cong \operatorname{Pic}(F M(R))$. This isomorphism can be described explicitly as follows. Let $A$ denote $F M(R)$, and let $e$ denote $e(1,1)$, the matrix idempotent with 1 in the $(1,1)$-entry and zeros elsewhere. Then the group isomorphism from Pic $(R)$ to Pic $(A)$ is given by $X \mapsto A e \otimes X \otimes e A$. Moreover, Ae $\otimes X \otimes e A \cong F M(X)$, the countably square matrices having at most finitely many nonzero entries, whose entries are elements of $X$.

Proof. By definition, for any ring $T, \operatorname{Pic}(T)$ denotes the group of autoequivalences of the category $T$-Mod. But $R$-Mod and $F M(R)-M o d=A$-Mod are equivalent categories by [3, p.14], hence have isomorphic Picard groups. Since $e A e \cong R$ and $A e A=A$, the map $X \mapsto A e \otimes X \otimes e A$ is an isomorphism of Picard groups.

It remains to prove that $A e \otimes X \otimes e A \cong F M(X)$. Define $\psi: A e \otimes X \otimes e A \rightarrow$ $F M(X)$ via $(z) \psi=[z]$, where the $(i, j)$-entry of $[z]$ is given by $e(i, i) \cdot z \cdot e(j, j)$. Here we identify $e(i, i) A e(1,1)$ with $R$, and, similarly, we identify $e(1,1) A e(j, j)$ with $R$.

It is straightforward to show that $\psi$ is a linear bijection; we need only show that $\psi$ is an $A-A$ bimodule map. To do this, it suffices to show $(e(k, l) z e(u, v)) \psi=$ $e(k, l)(z) \psi e(u, v)$. But $e(k, l) z e(u, v)$ consists of zeros off row $k$ and off column $v$; in fact, $e(k, l) z e(u, v)$ consists of the $(l, u)$-entry from $z$ in the $(k, v)$-position and zeros elsewhere. Similarly, $e(k, l)[z] e(u, v)$ has zero entries except in the $(k, v)$-position, which contains the $(l, u)$-entry of $z$. Thus, $[e(k, l) z e(u, v)]=e(k, l)[z] e(u, v)$, and so $\psi$ is a bimodule isomorphism.

A key ingredient in the proof of our main result is the fact, noted by Camillo in [6], that automorphisms of $B=R F M(R)$ behave nicely with respect to the subring $A=F M(R)$. We show now how Camillo's idea can be tailored to the situation at hand.

Lemma 2.6. Let $A$ be a ring with local units, and let $B=\operatorname{End}\left({ }_{A} A\right)$.

(1) Let $M$ be a left $A$-module. Then $M$ is finitely generated (in the categorical sense) if and only if there exist $x_{1}, \ldots, x_{n} \in M$ with $M=A x_{1}+\cdots+A x_{n}$. 
(2) Let $b \in B$. Then ${ }_{A} A b$ is contained in a finitely generated submodule of ${ }_{A} A$ if and only if $b \in B A$.

(3) Suppose that ${ }_{A} A e^{\tau}$ is finitely generated for each $\tau \in A u t(B)$ and each $e \in E$. Then $B_{\sigma} \in H$ for each $\sigma \in \operatorname{Aut}(B)$.

Proof. (1) The categorical definition of finitely generated is: for any collection $\left\{M_{\alpha}\right\}_{\alpha \in I}$ and for any surjection $f: \bigoplus_{\alpha \in I} M_{\alpha} \rightarrow M$ there is a finite subset $J=$ $\left\{\alpha_{1}, \ldots, \alpha_{n}\right\}$ of $I$ such that $\left.f\right|_{\oplus_{\beta \in J} M_{\beta}}$ is surjective. For any unitary module $M$, the map $g: \bigoplus_{x \in M} A \rightarrow M$ given by right multiplication by $x$ in the $x$-th coordinate is a surjection. The result is now clear.

$(2)(\Rightarrow)$ By part (1) we have $A b \subseteq A x_{1}+\cdots+A x_{n}$, where $x_{1}, \ldots, x_{n} \in A$. Then there exists $e \in A$ with $x_{i} e=x_{i}$ for all $i$. So for each $a b \in A b$ we have $a b e=a b$. But this yields that $b e=b$, since viewed as functions on $A$ these functions are equal for all $a \in A$. Thus $b \in B A$. $(\Leftarrow)$ We note that $b \in B A$ if and only if $b=b e$ for some $e \in E$; so upon writing $b=b e$ we get $A b=A b e \subseteq A e$; since $e \in A$ we have that ${ }_{A} A e$ is finitely generated.

(3) By Corollary 1.17 we need only show that $A \subseteq B \cdot A^{\sigma}$ and $A \subseteq B \cdot A^{\sigma^{-1}}$; this is equivalent to showing that $A^{\sigma^{-1}} \subseteq B A$ and $A^{\sigma} \subseteq B A$. But letting $\tau=\sigma$, from part (2) and the hypotheses we get that $e^{\sigma} \in B A$ for all $e \in E$, so that $a^{\sigma}=(a e)^{\sigma}$ (for some $e \in E)=a^{\sigma} e^{\sigma} \in B \cdot B A \subseteq B A$ for all $a \in A$. The proof that $A^{\sigma^{-1}} \subseteq B \cdot A$ is similar, letting $\tau=\sigma^{-1}$.

Proposition 2.7 (Camillo [6, p. 188]). Let $R$ be any unital ring, let $A=F M(R)$, and let $B=R F M(R)$. Let $E$ denote the set of local units of $A$ consisting of finite sums of the standard matrix units $\left\{e_{i i}\right\}_{i \in \mathbf{N}}$. Then for any $\tau \in A u t(B)$ and $e \in E$ we have that ${ }_{A} A e^{\tau}$ is finitely generated. In particular, $B_{\sigma} \in H$ for every $\sigma \in A u t(B)$.

Proof. Since any ${ }_{A} A e^{\tau}$ is a finite direct sum of left $A$-modules of the form ${ }_{A} A e_{i i}^{\tau}$, we need only show that each ${ }_{A} A e_{i i}^{\tau}$ is finitely generated. But ${ }_{A} A e_{11}^{\tau} \cong{ }_{A} A e_{i i}^{\tau}$ for all $i \geq 1$; the isomorphism is given by right multiplication by $e_{1 i}^{\tau}$, with right multiplication by $e_{i 1}^{\tau}$ as the inverse map. Thus it suffices to show that ${ }_{A} A e_{11}^{\tau}$ is finitely generated.

For each $a \in A$ we have $a e_{11}^{\tau}=\sum_{i=1}^{\infty} a e_{11}^{\tau} e_{i i}$, since $a e_{11}^{\tau} \in A$ means that the sum is actually finite. Thus, using the fact that $e_{11}^{\tau}$ is idempotent, we get $a e_{11}^{\tau}=$ $a e_{11}^{\tau} \cdot e_{11}^{\tau}=\sum_{i=1}^{\infty} a e_{11}^{\tau} e_{i i} e_{11}^{\tau}$.

We claim that ${ }_{A} A e_{11}^{\tau}$ is finitely generated if and only if $a e_{11}^{\tau} e_{i i} e_{11}^{\tau}=0$ for almost all integers $i$ and for all $a \in A$. To see this, suppose $A e_{11}^{\tau}$ is finitely generated. Then by Lemma 2.6(2) we have that $e_{11}^{\tau} \in B A$, so there exists a set of integers $\left\{i_{1}, \ldots, i_{n}\right\}$ such that $e_{11}^{\tau}=e_{11}^{\tau} \sum_{t=1}^{n} e_{i_{t} i_{t}}$. But then $e_{11}^{\tau} e_{i i}=0$ for all $i \notin\left\{i_{1}, \ldots, i_{n}\right\}$, and the result is clear. Conversely, we use the observation of the previous paragraph to get $a e_{11}^{\tau}=\sum_{i=1}^{\infty} a e_{11}^{\tau} e_{i i} e_{11}^{\tau}$ for each $a \in A$. So if terms of the form $a e_{11}^{\tau} e_{i i} e_{11}^{\tau}$ are zero for almost all $i$, then this equation says that the finite set $\left\{e_{j j} e_{11}^{\tau} \mid j \in \mathbf{N}\right.$ has $a e_{11}^{\tau} e_{j j} e_{11}^{\tau}$ nonzero for some $\left.a \in A\right\}$ generates $A e_{11}^{\tau}$.

Now define $h \in \operatorname{End}\left({ }_{A} A\right)=B=R F M(R)$ by setting, for each $a \in A$,

$$
(a) h=\sum_{k=1}^{\infty} a e_{11}^{\tau} e_{k k} e_{1 k}^{\tau} .
$$

This is well defined, since for each $a \in A$ the element $a e_{11}^{\tau} e_{k k}$ is nonzero for at most finitely many integers $k$. It is easy to show that $h$ is left $A$-linear, so that $h$ is indeed in $\operatorname{End}\left({ }_{A} A\right)$. In particular, we may apply $\tau^{-1}$ to $h$ to obtain the element 
$h^{\tau^{-1}}$ of $B$. Since $e_{11} \in A$ we have $e_{11} h^{\tau^{-1}} \in A$, so in particular $e_{11} h^{\tau^{-1}} e_{i i}=0$ for almost all $i$. Now apply $\tau$ to both sides to get $e_{11}^{\tau} h e_{i i}^{\tau}=0$ for almost all $i$; i.e., $a e_{11}^{\tau} h e_{i i}^{\tau}=0$ for almost all $i$, for all $a \in A$. Using the definition of $h$, this gives $\left(\sum_{k=1}^{\infty} a e_{11}^{\tau} \cdot e_{11}^{\tau} e_{k k} e_{1 k}^{\tau}\right) e_{i i}^{\tau}=\left(\sum_{k=1}^{\infty} a e_{11}^{\tau} e_{k k} e_{1 k}^{\tau}\right) e_{i i}^{\tau}=0$ for all $a \in A$, and for almost all $i$. But $e_{1 k}^{\tau} e_{i i}^{\tau}=e_{1 i}^{\tau}$ when $k=i$, and is zero otherwise. Using this, all terms but one in the sum become zero, and we get $a e_{11}^{\tau} e_{i i} e_{1 i}^{\tau}=0$ for all $a \in A$ and almost all $i$. Now multiplying both sides on the right by $e_{i 1}^{\tau}$ we get $a e_{11}^{\tau} e_{i i} e_{11}^{\tau}=0$ for all $a \in A$ and almost all $i$.

Therefore, by the claim established in the previous paragraph we conclude that $A e_{11}^{\tau}$ is finitely generated, which in turn yields the first statement of the proposition.

The second statement now follows immediately from Lemma 2.6(3).

Proposition 2.8. Let $R$ be a unital ring.

(1) $\operatorname{Pic}(F M(R))=J$.

(2) $\operatorname{Pic}(\operatorname{RFM}(R))=H$.

Proof. (1) Let $A$ denote $F M(R)$. By Proposition 2.5 it suffices to show that $F M(X) \in J$ for any $X \in \operatorname{Pic}(R)$. Since $R$ has an identity, $X \in \operatorname{Div}(R)$ and $R \in \operatorname{Div}(X)$. Thus, there exists an integer $n$ and split epimorphisms $\alpha: X^{n} \rightarrow R$ and $\beta: R^{n} \rightarrow X$. We can write $\alpha=\left[\alpha_{1}, \ldots, \alpha_{n}\right]^{T}$, where $\alpha_{i}: X \rightarrow R$; similarly, we can write $\beta=\left[\beta_{1}, \ldots, \beta_{n}\right]^{T}$, where $\beta_{i}: R \rightarrow X$.

We define $\hat{\alpha}: F M(X)^{n} \rightarrow A$ and $\hat{\beta}: A^{n} \rightarrow F M(X)$ as follows. Let $\hat{\alpha}_{i}$ be the scalar matrix with $\alpha_{i}$ on the diagonal. This is an infinite matrix. Define $\hat{\beta}_{i}$ similarly, and set $\hat{\alpha}=\left[\hat{\alpha_{1}}, \ldots, \hat{\alpha_{n}}\right]^{T}$ and $\hat{\beta}=\left[\hat{\beta_{1}}, \ldots, \hat{\beta_{n}}\right]^{T}$. It is easy to check that these are split $A$-epimorphisms, which yields $F M(X) \in J$.

(2) Let $B$ denote $\operatorname{RFM}(R)$, and let $X \in \operatorname{Pic}(B)$. By Corollary 2.4 we have $X \cong B_{\sigma}$ as bimodules for some $\sigma \in A u t(B)$. So it suffices to show that $B_{\sigma} \in H$. But this fact has been established in Proposition 2.7 above.

Theorem 2.9. For any unital ring $R$ we have isomorphisms of Picard groups

$$
\operatorname{Pic}(R) \cong \operatorname{Pic}(F M(R)) \cong \operatorname{Pic}(\operatorname{RFM}(R)) .
$$

Proof. The first isomorphism is given in Proposition 2.5. By the previous proposition we have $J=\operatorname{Pic}(F M(R))$ and $H=\operatorname{Pic}(R F M(R))$. Thus the second isomorphism follows from Theorem 1.14.

As one consequence of the previous theorem we conclude that for any ring $R$ there is a ring $B$ (namely, $B=R F M(R)$ ) for which $\operatorname{Pic}(B)$ is outer induced, and for which $\operatorname{Pic}(R) \cong \operatorname{Pic}(B)$. That the groups $\operatorname{Pic}(R)$ and $\operatorname{Pic}(R F M(R))$ are always isomorphic is perhaps surprising, as the rings $R$ and $R F M(R)$ are rarely Morita equivalent.

Using Theorem 2.9, we can now show that the group of outer automorphisms of $R F M(R)$ which fix $R$ elementwise is abelian when $R$ is commutative. This extends a result of Rosenberg and Zelinsky which first appeared in 1961; see [12].

Corollary 2.10. Let $R$ be a commutative unital ring. Let $O u t_{R}(R F M(R))$ denote the outer automorphisms of $R F M(R)$ which fix the scalar matrices of $R F M(R)$. Then $\mathrm{Out}_{R}(R F M(R))$ is an abelian group. In fact, Out $R(R F M(R)) \cong \operatorname{Picent}(R)$.

Proof. We start by noting that since $R$ is commutative, any inner automorphism of $\operatorname{RFM}(R)$ fixes the scalar matrices of $R F M(R)$; thus $\operatorname{Out}_{R}(R F M(R))$ is welldefined. For any ring $T$ with center $Z$ we define Picent $(T)=\{X \in \operatorname{Pic}(T) \mid z x=$ 
$x z$ for all $x \in X, z \in Z\}$. If $R$ is commutative then $\operatorname{Picent}(R)$ is an abelian group, since the map which takes $x \otimes y \in X \otimes_{R} Y$ to $y \otimes x \in Y \otimes_{R} X$ is easily shown to be an isomorphism (using the above definition, and the fact that $Z=R$ ). Thus it suffices to show that $\operatorname{Out}_{R}(\operatorname{RFM}(R)) \cong \operatorname{Picent}(R)$.

Let $X$ be an element of $\operatorname{Pic}(\operatorname{RFM}(R))$; so by Corollary 2.4 we have $X=$ $R F M(R)_{\alpha}$ for some $\alpha \in \operatorname{Aut}(R F M(R))$. We claim that $X \in \operatorname{Picent}(R F M(R))$ if and only if $\alpha$ fixes the scalar matrices of $\operatorname{RFM}(R)$; i.e., if and only if $\alpha$ generates an element of $\operatorname{Out}_{R}(R F M(R))$ inside $\operatorname{Out}(R F M(R))$. To see this, we note that the scalar matrices of $R F M(R)$ form the center of $R F M(R)$, and are isomorphic to $R$. Now if $X \in \operatorname{Picent}(R F M(R))$ and $r \in R$, then for all $x \in X$ we have $x * r=r x$, which gives $x r^{\alpha}=r x$. But since any ring automorphism preserves centers we get that $r^{\alpha}$ is in the center of $R F M(R)$, so $x r^{\alpha}=r^{\alpha} x$. Thus we get $r x=r^{\alpha} x$ for all $x \in X$, which gives $\left(r-r^{\alpha}\right) x=0$ for all $x \in X$, so that $r=r^{\alpha}$ as $X$ is faithful. The converse statement is proved in a similar way.

Now the isomorphism $\theta: \operatorname{Pic}(R) \rightarrow \operatorname{Pic}(\operatorname{RFM}(R))$ described subsequent to Example 2.11 is easily seen to take Picent $(R)$ to Picent $(R F M(R))$. But the above claim yields that $\operatorname{Picent}(R F M(R)) \cong \operatorname{Out}_{R}(R F M(R))$, and we are done.

In fact, Rosenberg and Zelinsky also show that $\mathrm{Out}_{R}\left(M_{n}(R)\right)$ has finite exponent dividing $n$. In contrast, however, an easy example shows that $\operatorname{Out}_{R}(R F M(R))$ can be torsion-free.

Example 2.11. Let $R$ be a Dedekind domain with Picent $(R) \cong \mathbf{Z}$ (the infinite cyclic group); such a domain exists by [7]. Now apply Corollary 2.10 to conclude that $\operatorname{Out}_{R}(R F M(R)) \cong \mathbf{Z}$, hence is torsion-free.

As yet another consequence of Theorem 2.9 we see that there is an isomorphism of groups $\theta: \operatorname{Pic}(R) \rightarrow \operatorname{Pic}(R F M(R))$, given by $X \mapsto \operatorname{Hom}_{A}(A, F M(X))$. (Here $A$ denotes $F M(R)$.) Furthermore, by Corollary 2.4 there is an isomorphism of groups $\tau: \operatorname{Out}(R F M(R)) \rightarrow \operatorname{Pic}(R F M(R))$. So

$$
\alpha=\tau \theta^{-1}: \operatorname{Out}(\operatorname{RFM}(R)) \rightarrow \operatorname{Pic}(R)
$$

is an isomorphism. For any integer $n$ we have the embedding $\beta: \operatorname{Out}\left(M_{n}(R)\right) \rightarrow$ $\operatorname{Out}(\operatorname{RFM}(R))$ induced by applying an element $\phi \in \operatorname{Aut}\left(M_{n}(R)\right)$ to each of the $n \times n$ blocks of $\operatorname{RFM}(R)$. Finally, for any integer $n$ we have the subgroup $I_{n}$ of $\operatorname{Pic}(R)$ described previously. A straightforward series of computations yields that, with $\alpha$ as defined above, the diagram described at the outset of this section indeed commutes. Thus our isomorphism in some sense provides a 'context' for the subgroups $I_{n}$ of $\operatorname{Pic}(R)$.

As mentioned above, $\operatorname{Pic}(R)$ is not in general the union of the subgroups $I_{n}$; rephrased, Out $(R F M(R))$ need not be the direct limit of the groups $\operatorname{Out}\left(M_{n}(R)\right)$. For instance,

Example 2.12. Let $k$ be the field of rationals, and let $R=M_{2}(k) \oplus k$ (ring direct sum). Then $R$ is semiperfect, so that $I_{n}=I_{1}$ for all $n \geq 1$, by [2, Theorem 27.11]. In fact, since $\operatorname{Aut}(k)$ is trivial it is easy to show that $\operatorname{Out}(R)$ is trivial, so that $\bigcup_{n} I_{n}=I_{1}$ is trivial as well. Thus in order to show that $\operatorname{Pic}(R) \neq \bigcup_{n} I_{n}$ we need only produce $P \in \operatorname{Pic}(R)$ having ${ }_{R} P$ not isomorphic to ${ }_{R} R$. Such a module is given by viewing $R$ as the subring $\left(\begin{array}{ccc}k & k & 0 \\ k & k & 0 \\ 0 & 0 & k\end{array}\right)$ of $M_{3}(k)$, and considering 
$P=\left(\begin{array}{ccc}0 & 0 & k \\ 0 & 0 & k \\ k & k & 0\end{array}\right)$. Then $P \in P i c(R)$ as $P \otimes P \cong R$, but ${ }_{R} P$ is not isomorphic to $R$ by a dimension argument.

It would be interesting to classify those rings $R$ for which $\operatorname{Pic}(R)=\bigcup_{n} I_{n}$.

\section{REFERENCES}

1. G. Abrams and J. Haefner, Bounded Picard groups, Colloq. Math. 72(2) 1997, 325-334. MR 97m:16003

2. F.W. Anderson and K.R. Fuller, 'Rings and Categories of Modules', 2nd ed., Graduate Texts in Mathematics (Volume 13), Springer Verlag, 1992. MR 94i:16001

3. P.N. Ánh and L. Márki, Morita equivalence for rings without identity, Tsukuba J. Math 11(1) 1987, 1-16. MR 88h:16054

4. M. Beattie and A. del Río, The Picard group of a category of graded modules, Comm. Algebra 24 1996, 4397-4414. MR 97j:16058

5. M. Bolla, Isomorphisms between endomorphism rings of progenerators, J. Algebra 871984 , 261-281. MR 85e:16052

6. V. Camillo, Morita equivalence and infinite matrix rings, Proc. A.M.S. 90(2) 1984, 186-188. MR 85a: 16045

7. L. Claborn, Every abelian group is a class group, Pac. J. Math 18 1966, 219-225. MR 33:4085

8. C.W. Curtis and I. Reiner, 'Methods of Representation Theory with Applications to Finite Groups and Orders (Volume II)', Pure and Applied Mathematics, Wiley Interscience, 1987. MR 88f: 16002

9. R. Gilmer, A note on generating sets for invertible ideals, Proc. A.M.S. 22 1969, 426-427. MR 38:2739

10. R. Guralnick, The genus of a module II. Roiter's theorem, power cancellation, and extension of scalars, J. Number Theory 26(2) 1987, 149-165. MR 88j:16008

11. R. Guralnick and S. Montgomery, On invertible bimodules and automorphisms of noncommutative rings, Trans. A.M.S. 341(2) 1994, 917-937. MR 94d:16027

12. A. Rosenberg and D. Zelinsky, Automorphisms of separable algebras, Pacific J. Math. 11 1961, 1109-1117. MR 26:6215

Department of Mathematics, University of Colorado, Colorado Springs, Colorado 80933

E-mail address: abrams@math.uccs.edu

E-mail address: haefner@math.uccs.edu 